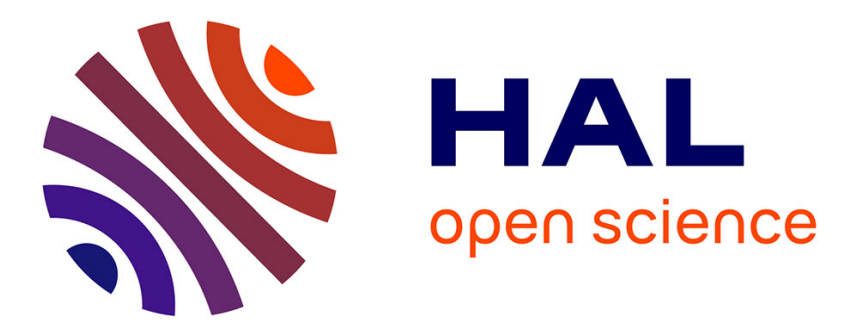

\title{
Multiphase Pipe Flow velocity profile measurements by Doppler Ultrasound Containing a High Level of Colored Noise
}

Jean-Pierre Remenieras, Denis Kouamé, Jean-Marc Grégoire, P. Jaraczewski, Frédéric Patat

\section{To cite this version:}

Jean-Pierre Remenieras, Denis Kouamé, Jean-Marc Grégoire, P. Jaraczewski, Frédéric Patat. Multiphase Pipe Flow velocity profile measurements by Doppler Ultrasound Containing a High Level of Colored Noise. IEEE International Ultrasonics Symposium, Nov 1996, San Antonio, Texas, United States. pp.629-634, 10.1109/ULTSYM.1996.584055 . hal-03153382

\section{HAL Id: hal-03153382 \\ https://hal.science/hal-03153382}

Submitted on 26 Feb 2021

HAL is a multi-disciplinary open access archive for the deposit and dissemination of scientific research documents, whether they are published or not. The documents may come from teaching and research institutions in France or abroad, or from public or private research centers.
L'archive ouverte pluridisciplinaire HAL, est destinée au dépôt et à la diffusion de documents scientifiques de niveau recherche, publiés ou non, émanant des établissements d'enseignement et de recherche français ou étrangers, des laboratoires publics ou privés. 


\title{
Multiphase Pipe Flow Velocity Profile Measurements By Doppler Ultrasound Containing A High Level Of Colored Noise
}

\author{
J.P.Remenieras, D.Kouamé, J.M.Gregoire, P.Jaraczewski, F.Patat,
}

LUSSI / GIP Ultrasons, 2 bis boulevard Tonnellé, BP 3223, 37032 Tours Cedex, France.

\begin{abstract}
This paper presents an application of continuous wave Doppler ultrasound measurement to two phases flow in pipes. A specific Doppler System has been developed for this purpose. The presence of a strong colored noise in the experimental Doppler signals was an incentive motivation to use a complex $U D V^{H}$ factored form of the Instrumental Variable identification technique. Direct Doppler frequency estimation is then obtained using the argument poles of the autoregressive model which identifies the Doppler part of the signal. The performed tests with complex parametric theoretical model, simulated and experimental Doppler signal have demonstrated the superiority of this adaptive signal processing method compared with the $U D U^{H}$ factored form of the Recursive Least Square autoregressive identification algorithm.
\end{abstract}

\section{INTRODUCTION}

The ultrasonic Doppler velocimeter has been used extensively in transcutaneous measurement of the blood velocity in human body. Although the principle of Doppler measurement is simple, the spectral content of analytic signals is complicated by a number of device related factors which introduce uncertainties in the velocity estimation. Low spatial resolution in region of large velocity gradients, low temporal resolution for rapidly changing flows, time transit and geometrica! effects, instrument and measurement noises cause broadening of the Doppler power spectra. [1-3]. Such broadening may also arise from limitation of the employed spectrum estimator [4-5]. Different Doppler frequency estimators can be used from the Doppler power spectral density (maximum amplitude detection, first moment, or parametric filters poles frequency estimators), and the performances of these estimators depend on the physical parameters of the Doppler signal (bandwidth, angle, SNR).

This paper presents an industrial application of continuous waves Doppler ultrasound velocity measurements to two phases flow in pipes. In many petroleum wells, the multiphase flow is stratified in two separated phases: the first stratum is a liquid phase (oil), the second is a gas phase with small scatters (oil particles). In order to measure the two phases velocity profiles, a specific instrument was developed and is described in $\$ 2$. In $\$ 3$, experimental signals obtained on a two phases loop are presented. Spectral analysis shows the presence of a strong level of centered and colored noise, superimposed on the Doppler signal. In most experimental cases, the bandwidth of this noise is much greater than the quarter of the sample frequency. This colored noise introduces an important bjas in classical frequency estimators. In $\$ 4$, a complex augmented $U D V^{H}$ identification factored form of the Instrumental Variable identification technique is presented as an alternative for estimating the Doppler frequency. Direct Doppler frequency estimation is then obtained using the argument poles of the autoregressive model which identifies the Doppler part of the signal. The results of this digital signal processing method are compared with the classical $U D U^{H}$ Recursive Least Square identification technique on a ARX model. $\$ 5$ compares the performances of the JV, RLS, and temporal autocorrelation algorithms on simulated Doppler signals with a strong level of colored noise. \$6 shows the velocity estimation on experimental Doppler signals.

\section{DESCRIPTION OF THE MEASUREMENT SYSTEM}

In the flowmeter, two stratified phases are found. The lower one is a liquid phase, and the upper one a gas phase. The measurement system consists in two sets of ultrasonic sensors. Each set is composed of eight identical and independent sensors located on a vertical line along both sides of the rectangular cross-section of pipe. The first set is adapted to measurements in the liquid phase and the second set to the gas. The ultrasound frequency emissions is respectively $750 \mathrm{KHz}$ and $2.25 \mathrm{MHz}$. The transducers angles are $45^{\circ}$. This arrangement allows the velocity profile to be obtained over the test section. Custom made electronics control sensor multiplexing, complex synchronous demodulation and signal acquisition by the computer.

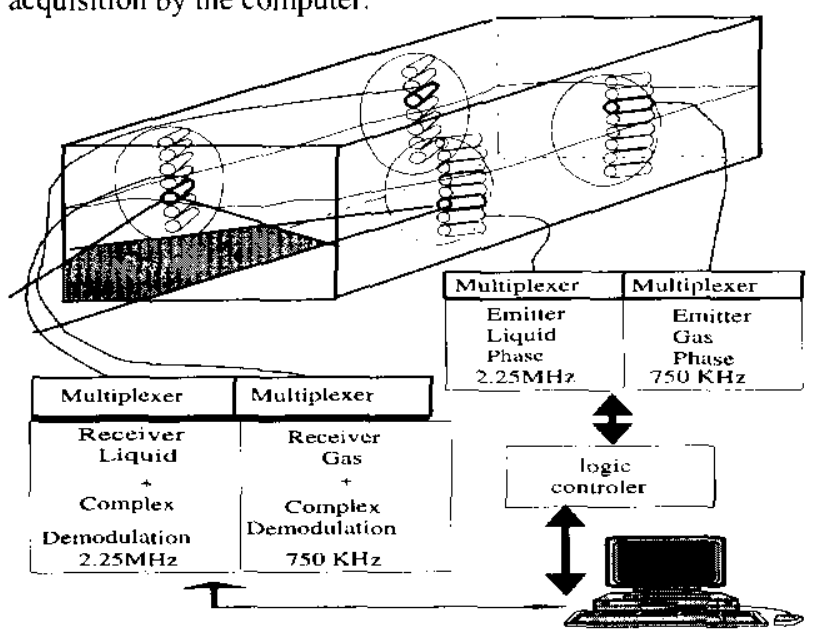

figure l: experimental measurement system 


\section{EXPERIMENTAL DOPPLER SIGNAL}

Figure 2 shows a typical real part of the complex Doppler signal in the liquid phase.

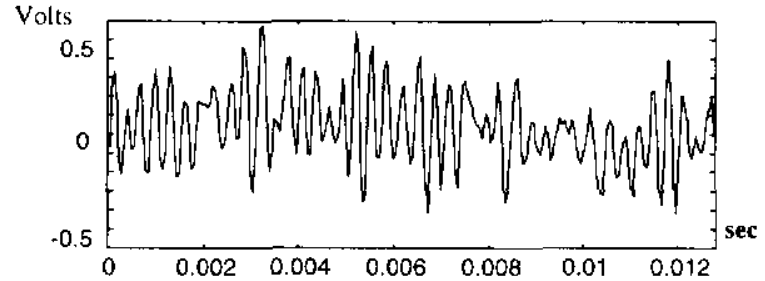

Figure 2: real part of the liquid complex demodulated Doppler signal

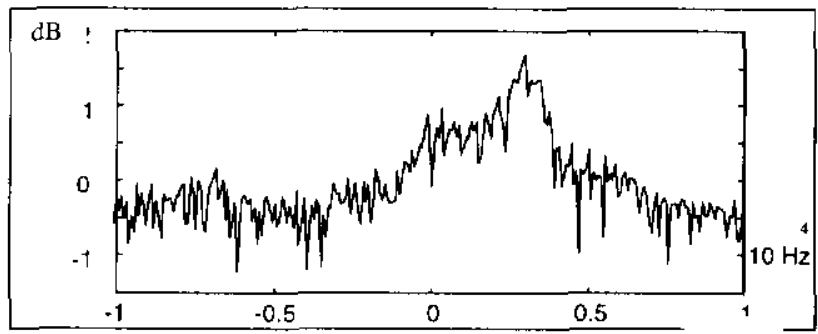

figure 3: PSD of the received signal on 1024 points FFT

Figure 3 shows the Power Spectral Density of the complex demodulated liquid signal received by the transducer. The Doppler frequency is about $3 \mathrm{KHz}$, and a low frequency noise is present around zero frequency. This noise is symmetrical, and then not linked to flow information. This phase noise can be produced by the wide-band structural vibrations which cause direct acoustical path modulation between the emitter and receiver transducers.

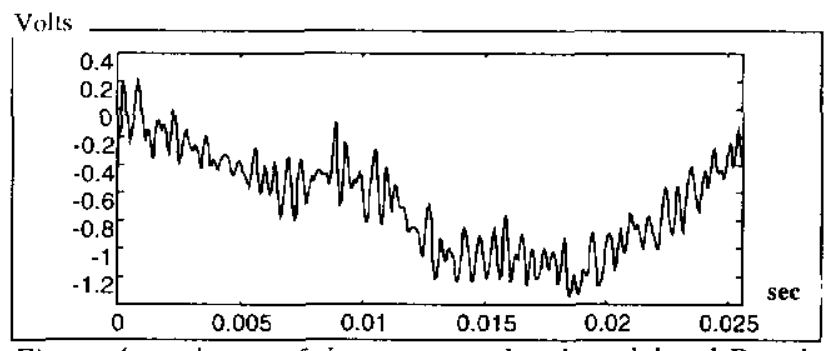

Figure 4: real part of the gas complex demodulated Doppler signal

Figure 4 shows the real part of the complex Doppler signal in the gas phase. Figure 5 shows the PSD of this signal. In that case the low frequency noise is much more important than for the liquid phase.

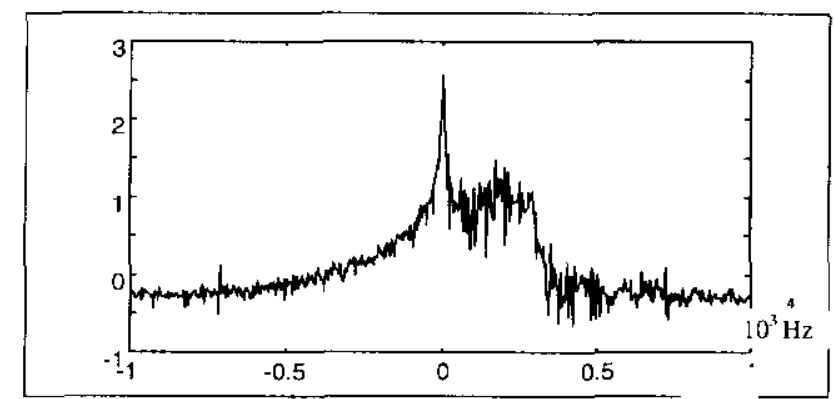

figure 5: PSD on 1024 points FFT of the received signal

\section{DOPPLER FREQUENCY ESTIMATION}

The classical parametric approach assumes that the added noise is white. J.Y.David and S.A.Jones [5] show that if the order of the autoregressive filter is adapted to the signal-tonoise ratio, the Doppler frequency obtained by the maximum of the spectral amplitude gives a better precision than the non parametric frequency estimation. When the signal-to-noise ration is high $(30 d B)$, a low autoregressive order ( 2 or 3 ) is necessary to minimize spectral line splitting which increases the variance of the frequency estimation. When the $S N R$ is low $(-10 d B)$, the same precision can be obtained with a higher order (5 or 6). In parametric approach, accurate Doppler frequency estimation is linked to correct parametric identification of the complex signal.

Since spectrum analysis shows the presence of a strong level of pink noise, a parametric technique based on the instrumental variable [7-8] algorithm is proposed. This technique was developed to solve the problem of parameter estimation for system with colored noise in which commonly used recursive least squares algorithm give biased estimation. In order to cope with the nonstationarity of the Doppler signal caused by rapidly changing flows, this algorithm is used in a time adaptive form with a forgetting factor. In order to adapt correctly the order of the parametric filter for a varying SNR, a complex UDV ${ }^{H}$ factorization technique is used to reformulate the conventional instrumental variable method. This technique simultaneously produced the parameter estimations and loss functions for all model orders, from one to the user specified number $p$ with approximately the same computational effort as the conventional IV algorithm. The correct order is then selected from a test on the diagonal elements of the D matrix.

Let us first look at the classical expression of the IV algorithm. Assuming that the process model can be represented by the difference equation model:

$$
z(t)=-a_{1} z(t-1)-\ldots-a_{n} z(t-n)+b u(t-1)+\ldots+b_{n} x(t-n)+\xi(t)
$$

Or in a more compact form:

$$
\begin{gathered}
z(t)=h^{T}(t) \theta_{O}+\xi(t) \\
\text { where } \theta_{0}=\left[a_{n}, b_{n}, \ldots, a_{2}, b_{2}, a_{1}, b_{1}\right]^{T} \\
\text { and } h(t)=[-z(t-n), u(t-n), \ldots,-z(t-1), u(t-1)]^{2}
\end{gathered}
$$

where $u(t)$ and $z(t)$ are the observed input and output sequences, $a_{i}$ and $b_{i}(\mathrm{i}=1,2, \ldots, \mathrm{n})$ are the model parameters and $\xi(t)$ is the noise process sequence which can be either white or colored. This model is called the process model. The instrumental variable vector $\zeta(t)$ and the information matrix $\mathrm{P}$ are defined by :

$$
\zeta(t)=[-x(t-n), u(t-n), \ldots,-x(t-2) ; u(t-2),-x(t-1), u(t-1)]^{T}
$$




$$
P=\left[\sum_{j=1}^{1} r^{i-j} \zeta(j) \cdot h^{T}(j)\right]_{d * d}^{-1} .
$$

where $r$ is the forgetting factor and $d=2 * n$. The IV estimate of the model parameters is then given by

$$
\hat{\theta}(t)=\left[\sum_{j=1}^{1} r^{-j} \zeta(t) \cdot h^{T}(t)\right]^{-1} \sum_{j=1}^{t} r^{t-j} \varsigma(t) \cdot z(t)(4.6)
$$

where $\hat{\theta}(t)$ is a consistent estimate of the model parameter $\theta_{0}$ under the conditions:

$\mathrm{E}\left\{r^{t-i} \varsigma(t), \xi(t)\right\}=0:$ The instrument is uncorrelated with the noise

$\lim \frac{1}{t} \sum_{j=1}^{t} r^{t-j} \zeta(j) \cdot h^{\tau}(j)$ has full rank

The instrument $x(t)$ can be selected in many differents ways [8]. The most widely used instruments are calculated using the regression model:

$$
x(t)=\zeta^{T}(t) \vartheta_{0}
$$

where $\vartheta_{0}$ is the latest available parameter estimate from the previous time of the process model. Another possibility is to choose the instrument $x(t)$ by the delayed $d$ output and input.

$$
x(t)=[-z(t-d-n), u(t-n), \ldots,-z(t-d-1), u(t-1)]^{T}
$$

In Doppler frequency estimation, input $u(t)$ of the parametric system is unknown so the data vectors $h(t)$ and $\zeta(t)$ are only formed with the output data.

$$
\begin{gathered}
h(t)=[-z(t-n), \ldots,-z(t-1)]^{T} \\
\varsigma(t)=[-x(t-n), \ldots,-x(t-2),-x(t-1)]^{T}
\end{gathered}
$$

The recursive IV algorithm can then be summarized by:

$$
\begin{aligned}
& S(t)=r+h^{T}(t) \cdot P(t-1) \zeta^{*}(t) \\
& K(t)=\frac{P(t-1) \cdot \zeta^{*}(t)}{S(t)} \\
& P(t)=\left[P(t-1)-\frac{P(t-1) \cdot \zeta^{*}(t) \cdot h^{T}(t) \cdot P(t-1)}{S(t)}\right] \frac{1}{r} \\
& \hat{\theta}(t)=\hat{\theta}(t-1)+K(t) \cdot\left(z(t)-h^{T}(t) \cdot \hat{\theta}(t-1)\right)
\end{aligned}
$$

where $\mathrm{K}(\mathrm{t})$ is the Kalman gain vector. This gain, with the innovation sequence updates the model parameters.

\section{The $U D V^{H}$ complex factored form of the IV algorithm:}

This form is a complex adapted form of the recursive real one given by Niu et Fisher [7]. The model is represented by the expression (4.1). By defining an augmented data vector $\varphi(t)$

$$
\varphi(t)=\left[h^{T}(t),-z(t)\right]^{T}
$$

and a augmented instrumental variable vector:

$$
\eta(t)=[\zeta(t),-x(t)]^{T}
$$

a new matrix called information accumulation matrix can be defined:

$$
C(t)=\left[\sum_{j=1}^{t} \eta(j) \cdot \varphi^{T}(j)\right]_{d \times d}^{-1}
$$

In this case, $d=2 n+1$. Since $\mathrm{C}(\mathrm{t})$ is a non symmetrical matrix, Bierman $U D U^{H}$ factorization technique used for RLS identification [9] cannot be used directly. The $U D V^{H} I V$ complex decomposition, which is a complex derived version of the Bierman algorithm, can be used to decompose the matrix $C(t)$.

$$
C(t)=\left[\sum_{j=1}^{1} \eta(j) \cdot \varphi^{T}(j)\right]_{d \times d}^{-1}=\left[C^{-1}(t-1)+\eta(t) \cdot \varphi^{T}(t)\right]_{d \times d}^{-1}
$$

With the rank one update relation, and by defining $f_{M}=U^{T}(t-1) \varphi(t), \quad g_{M}=D(t-1) \cdot f_{M}^{*}$ for the model and $f_{I}=V^{T}(t-1) \eta(t), g_{l}=D(t-1) . f_{i}^{*}$ for the instrument, the $C(t)$ matrix can be expressed:

$C(t)=U(t) D(t) V^{H}(t)=U(t-1)\left[D(t-1)-\frac{g_{r} g_{M}^{H}}{\beta(t)}\right] V^{H}(t-1)$

with $\beta(t)=1+f_{M}^{T} g$, and $g_{M}^{H}$ the hermitic conjugate of $g_{M}$. The bracketed part of $C(t)$ is decomposed through a series of orthogonal transformation to obtain:

$$
\begin{array}{r}
D(t-1)-\frac{g_{t} g_{M}{ }^{H}}{\beta(t)}=\bar{U}(t) \bar{D}(t) \bar{V}^{H}(t) \\
C(t)=U(t-1) \bar{U}(t) \cdot \bar{D}(t) \cdot(V(t-1) \bar{V}(t))^{H}
\end{array}
$$

This relation updates recursively the $U(t-1)$ and $V(t-1)$ matrix to obtain $U(t)$ and $V(t)$. It can be showed [7] that with this augmented structure, a multiple model structure is produced. The model parameters are calculated in the above diagonal of the odd-numbered columns in $\mathrm{U}(\mathrm{t})$. The parameters of the auxiliary model are available in $V(t)$. The inverse of the generalized loss function $J$ are available in the diagonal of the $\mathrm{D}(\mathrm{t})$ matrix.

$$
J=\sum_{j=1}^{t}\left[(x(j)-\hat{x}(j))\left(z(j)-h^{T}(j) \hat{\theta}(j)\right)\right]
$$

In the expression (4.13), a forgetting factor $\mathrm{r}$ can be introduced to track the non stationarities of the signal. The $U D U^{\prime \prime}$ RLS factorization is a complex adapted form of the recursive real one given in [6].

\section{Simulation example}

The $U D V^{H}$ VI and $U D U^{H}$ RLS algorithms are compared on an autoregressive model AR2 with a colored noise $x$ si( $(t)$ which is a Moving Average filtering of the noise $\mathrm{e}(\mathrm{t})$. The complex system is represented by:

$z(t)=(1+i) z(t-1)-(0.0625+0.5 i) z(t-2)+x \operatorname{si}(t)$

with $x$ si $(t)=-3 e(t)-5 e(t-1)+20 e(t-2)$, where $e(t)$ is a complex white noise. The $U$ and $V$ matrix are initialized as the identity matrix $\mathrm{I}$ and $D=\lambda \mathrm{I}$ with $\lambda=10^{3}$. 


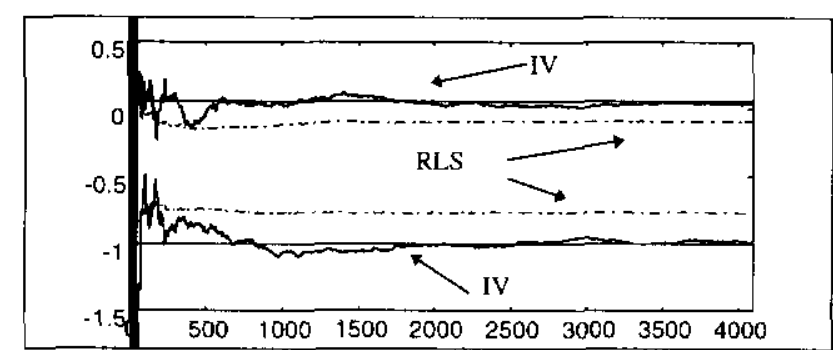

figure 6: Real part of the $a_{1}$ and $\mathrm{a}_{2}$ parameters.

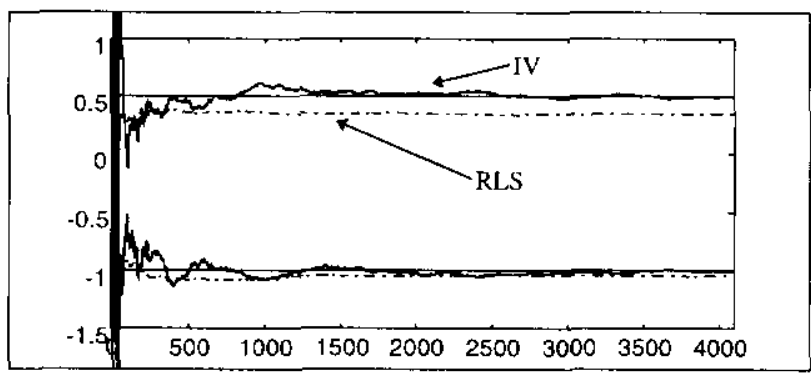

figure 7: Imaginary part of the $a_{1}$ and $\mathrm{a}_{2}$ parameters.

Figures 6 and 7 show the real and imaginary part of the parameters obtained by the $U D V^{H} \mathrm{IV}$ and $U D U^{H} \mathrm{RLS}$ recursive identification algorithms.

One can see in this numerical example, that the $U D U^{H}$ RLS algorithm gives a biased autoregressive estimate in presence of colored noise. The $U D V^{H} I V$ gives a better precision in the parameters estimation. When the $a_{i}$ parameters are calculated, the Power Spectral Density $P(f)$ is classically obtained by:

$$
\begin{aligned}
P(f) & =\frac{\gamma}{\mid \sum_{k=1}^{p} a[k] \exp \left(-\left.2 \pi j k f T_{k}\right|^{2}\right.}=\frac{\gamma}{\left|\sum_{k=1}^{p} a[k] z^{-k}\right|_{z=\exp \left(2 \pi j T_{r}\right)}^{2}} \\
& =\frac{\gamma}{\left|\prod_{k=1}^{n}\left(z-z_{k}\right)\right|_{z=\exp \left(-2 \pi i T_{r}\right)}^{\left.\right|^{2}}}
\end{aligned}
$$

$\gamma$ is a constant depending on the covariance of the modeling error, $z_{k}$ are the roots of the denominator of $(4,16)$. Thus, if the logarithm of $P(f)$ is taken, the PSD is the sum of first order elements. The " $k$ " peak frequencies $f_{p r e k}[k]$ are directly expressed by:

$$
f_{p \text { rad }}[k]=\frac{f_{c}}{2 \pi} \operatorname{Arg}\left(z_{k}\right)
$$

which has the same form as the autocorrelation Doppler frequency estimator:

$$
f_{K}=\frac{f_{\varepsilon}}{2 \pi} \operatorname{Arg}\left(\Re_{z z}\left(T_{\epsilon}\right)\right) .
$$

5. COMParison OF THE $U D V^{H}$ IV AND $U D U^{H}$ RLS ALGORITHM ON SIMULATED DOPPLER SIGNAL WITH A STRONG LEVEL OF COLORED NOISE

For these simulation, the simulation proposed by Jones and Giddens [2], which takes into account the time transit effects in the Doppler signal, was used. The flow velocity is $\mathrm{V}=1 \mathrm{~m} / \mathrm{s}, \theta=45^{\circ}$, the sampling frequency $f_{c}=20 \mathrm{KHz}$, the emission frequency $f_{0}=2.25 \mathrm{MHz}$, and the width of the measured volume $l=6.10^{-3} \mathrm{~m}$. The "noisy" Doppler signal is obtained by adding to the simulated Doppler signal $z_{T T}$ an AR2 centered noise $x$ si(t). The two poles of this AR model are real, so the maximum amplitude frequency of the noise DSP is zero. The SNR is determined by the ratio of the variance of the white noise $\sigma_{D}$ which is used to model the stochastic Doppler signal and the variance $\sigma_{e}$ of the white noise e(t) which drives the AR process. Figure 8 shows the real and imaginary parts of the simulated Doppler signal and Figure 9 shows the PSD with $S N R=O d B$.

$$
\begin{gathered}
z_{D O P}(t)=z_{T T}(t)+x \operatorname{si}(t) \\
\text { with } x \operatorname{si}(t)=x \operatorname{si}(t-1)-0.21 . x \operatorname{si}(t-2)+e(t)
\end{gathered}
$$

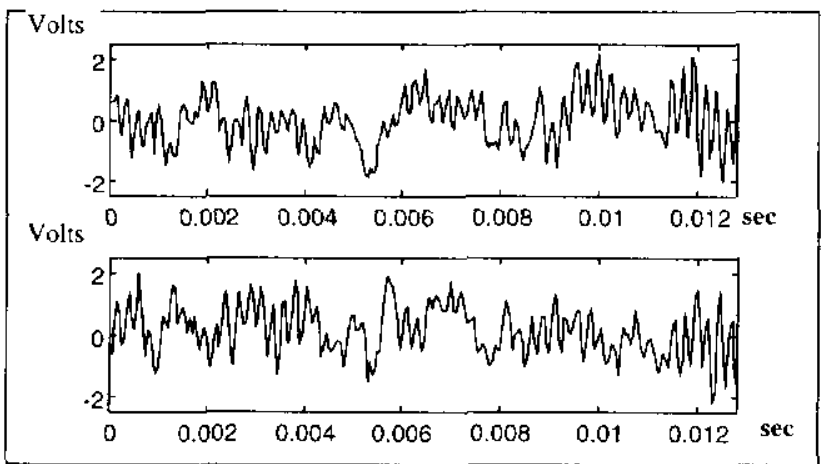

figure 8: real and imaginary part of the complex simulated Doppler Signal

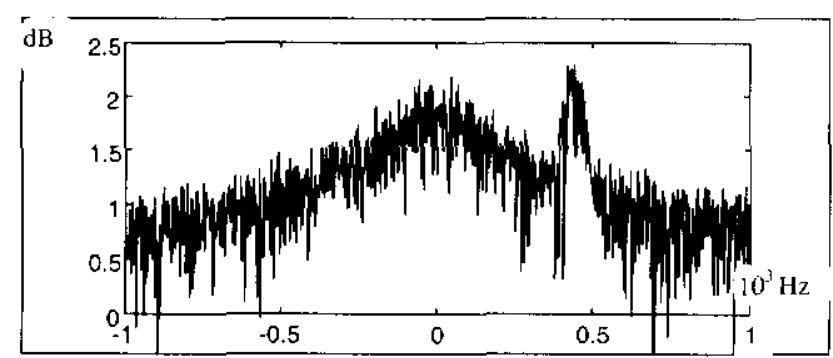

figure 9: PSD of the complex simulated Doppler signal on 1024 points $F F T$

The recursive $U D V^{H}$ IV and $U D U^{H}$ RLS algorithm where applied to this signal. The real and imaginary parts of the parameters are showed in figure 10 . The order is determined by inspecting the inverse of the D matrix for the two algorithms (for the IV algorithm, the norm of $J$ is taken because the generalized loss function is complex). We plot on figure 10 the elements of the diagonal of the norm of the inverse of $\mathrm{D}$. The minimum of $J^{(n)}$ is obtained for the order 3. 


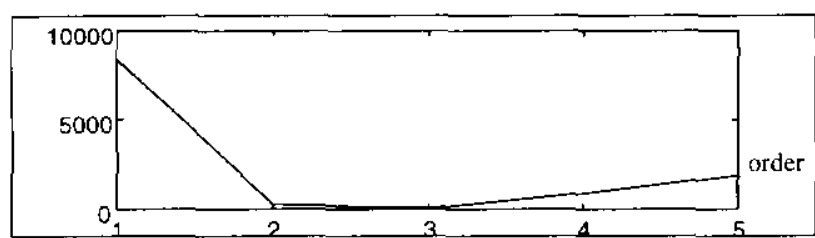

figure 10: Norm of the generalized loss function for the UDV "IV algorithm for different onder.

We plot on figure 11 the elements of the diagonal of the inverse of D. The loss function is stabilized at its minimum for the model order 4 .

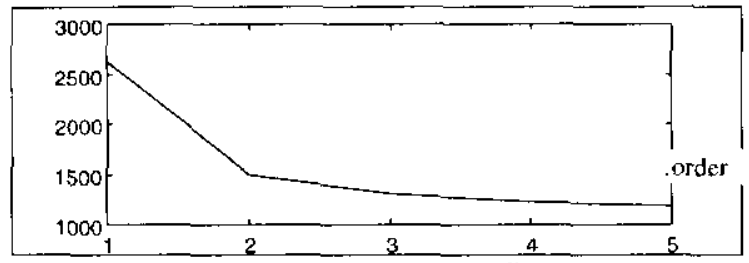

figure 1/: Loss function for the UDU ${ }^{H} R L S$ algorithm.

The trajectories of the real part of the parameters ars plotted in figure 12 . We cannot see significant difference between two parameters $(0.6$ and 0$)$, and a few differences between one ( -1 for the IV and -1.5 for the RLS).

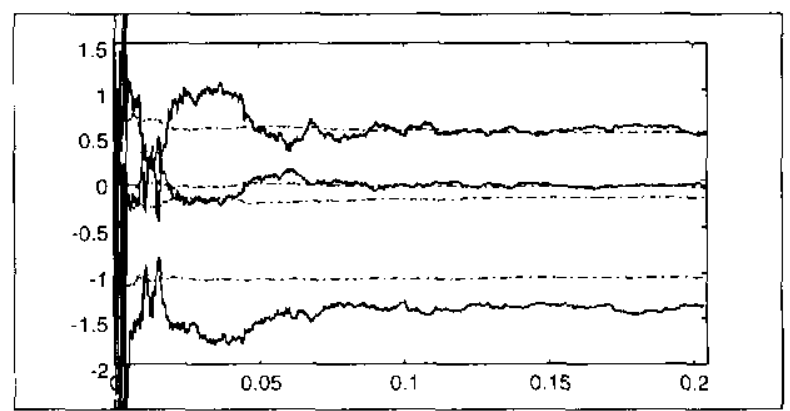

figure 12: real part of the identification on modeled Doppler signal

With these parameters, we can compute on figure 13 the PSD IV order 3 and PSD AR order 4 . We can clearly see the position of the Doppler signal. The maximum amplitude is given by the pole decomposition and is situate neat $4.4 \mathrm{KHz}$. The strong colored noise is centered round zero frequency. The ratio between the doppler and the noise maximum amplitude is bigger for the IV algorithm.

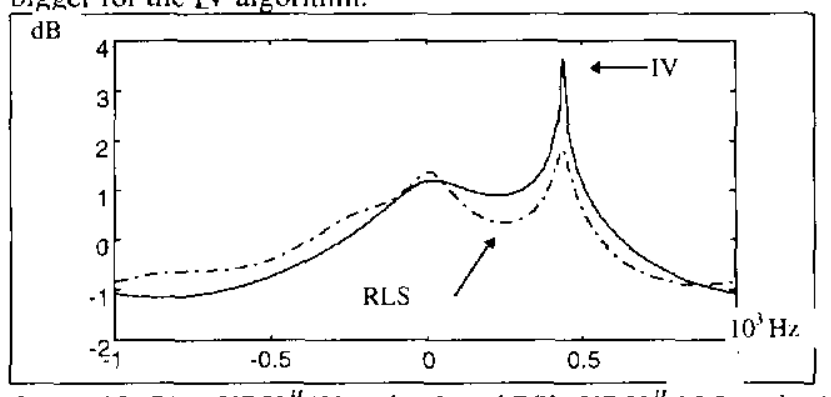

figure 13: PSD UDV "IV order 3 and PSD UDU ${ }^{\prime \prime} R L S$ order4
Finally, the Doppler frequency estimation can be computed by the relation (4.17). It can be seen on the figure 14 , that the two techniques give similar result. The small difference in the parameters estimate, do not seem to have an important influence on the position of the poles. The two techniques give a very good estimate of the Doppler frequency. The Doppler frequency estimation on the bloc of 4096 points by the argument of the autocorrelation function is $f_{K}=1970 \mathrm{~Hz}$. In colored noise, this estimator is strongly biased and gives a frequency estimate with more than $50 \%$ of error.

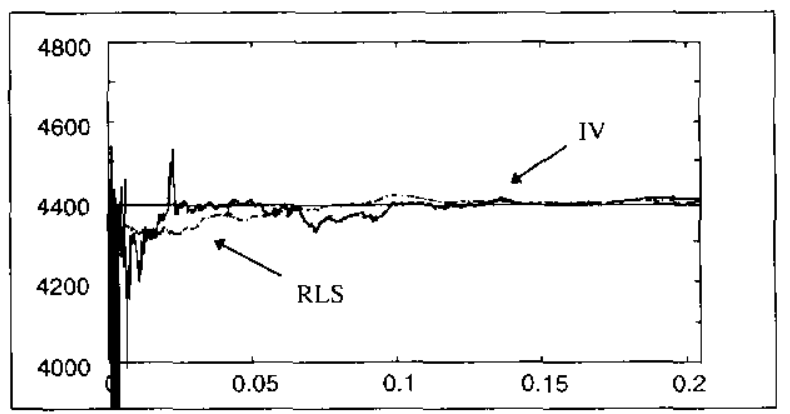

figure 13: Doppler frequency estimate by the pole of the $U D V^{H} I V$ order 3 and $U D U^{H} R L S$ order 4 algorithm.

\section{VEJOCITY ESTIMATION ON EXPERIMENTAL DOPPLER SIGNAL}

In the experimental system, we estimated the Doppler frequency in the gas phase. We select only a paire of transducers and apply the $U D V^{\prime \prime} I V, U D U^{\prime \prime} R L S$ and $f_{K}$ algorithm. The diagonals matrix give us the order 2 for the two algorithm. The PSD obtained by the two algorithms are ploted in figure 14. Like in the simulated Doppler case, the experimental Doppler peak obtain by the IV algorithm is higher than the RLS one. The frequency estimation by the pole method is plotted as function of time in figure 15. The difference between the two frequencies is $25 \mathrm{~Hz}$ and represent $1.1 \%$ of the measured frequency. The frequency estimation obtained by the correlation angle algorithm on the same 4096 points data bloc is $38.1 \mathrm{~Hz}$. This signal processing technique is non adapted to this noisy signal

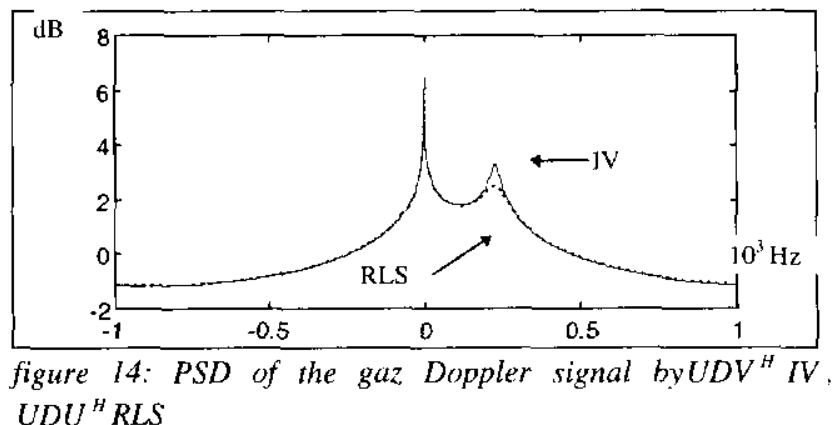
$U D U^{H} R L S$ 


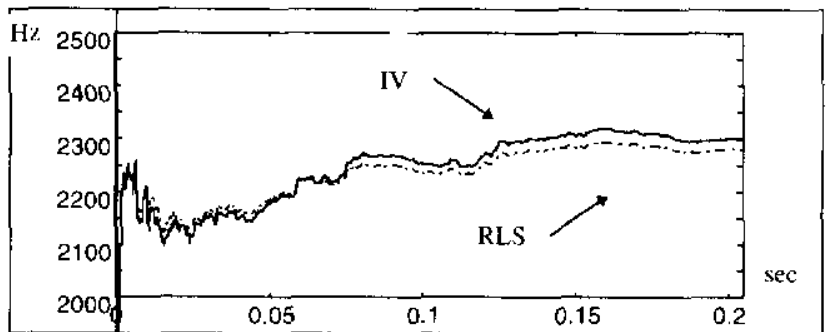

figure 15: Doppler frequency estimation in gaz flow

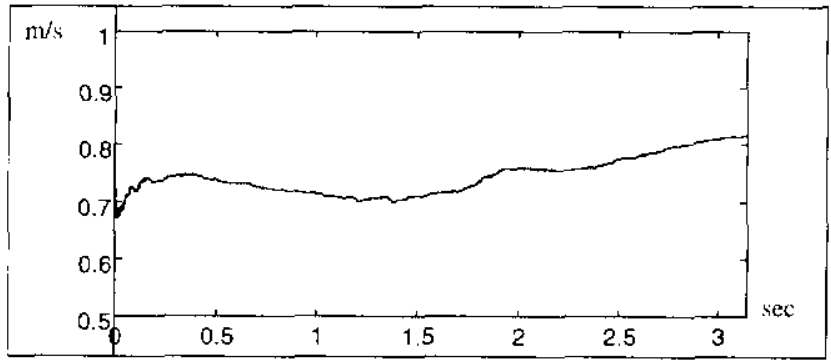

Figure 16: Velocity estimation in gaz flow with the $U D V " I V$ algorithm during 3.5 seconds. $r=0.99$.

In the liquid phase, with a pulse flow, the frequency estimation during 7.3 second is plot on figure 17 .

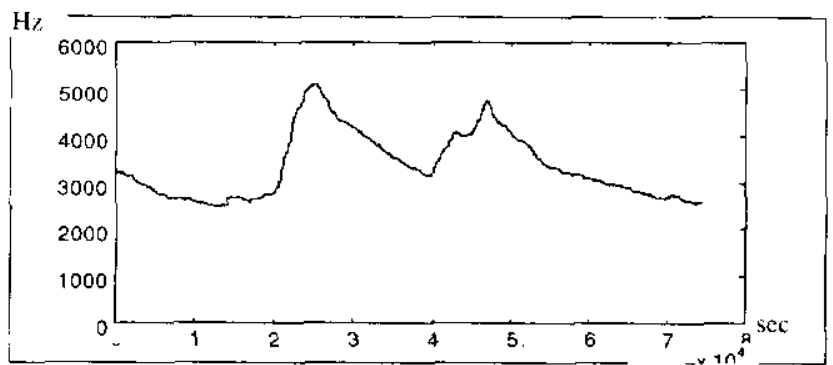

Figure 17: Doppler frequency estimation in liquid pulse flow with the $U D V^{H} I V$ algorithm. $r=0.99$.

\section{Conclusion}

An industrial application of CW Doppler ultrasound velocity measurements to two phased flow shows the presence of a strong colored noise. A specific IV identification algorithm has been adapted in complex form to be used on the analytic Doppler signal. Comparison with $U D U^{H}$ recursive least square AR identification shows that the parameters estimated with the IV algorithm are not biased by the colored noise. The two techniques are used in adaptive form to take into account the non stationarity of the flow. The triangular decomposition of the data matrix allows to adapt the model order to the varying Doppler SNR. The Doppler frequency estimation is obtained by the argument of the pole which identifies the Doppler signal. The diagonal matrix in the triangular decomposition allows to estimate the order of the model which gives the best Doppler frequency estimation. If the two algorithms orders are correctly chosen, the two frequency estimators obtained by the two methods on theoretical and experimental Doppler signals give nearly the same Doppler frequency estimation and are very close to the true frequency. Velocity measurements on liquid and gas flows have demonstrate the feasibility of the measurement principle associated with this adapted signal processing.

[1] J.L.Garbini, F.K.Forster, J.E.Jorgensen, "Measurement of fluid turbulence based on pulsed ultrasound techniques. Part 1\&2", J.Fuid Mech. vol 118, pp.445-470, 1982.

[2] S.A.Jones and D.P.Giddens, "A simulation of transit time effects in Doppler ultrasound signal", Ultrasound in Med\&Biol. Vol.16, No6, pp607-619, 1990.

[3] D.Censor, VL.Newwhouse, T.Vontz, H.V.Ortega, "Theory of ultrasound Doppler Spectra Velocimetry for Arbitrary Beam and Flow Configurations", IEEE Trans, on Biomedical Engineering, vol 35, no9, 1988.

[4] P.J Vaitkus and R.S.C.Cobbold, "A comparative study and assesment of Doppler ultrasound spectral estimation techniques, Part 1\&2:,"Ultrasound Med.Biol.,vol14, pp.661672,1988 .

[5] J.Y.David, S.A.Jones, D.P.Giddens "Modern spectral analysis techniques for blood flow velocity and spectral measurements with pulsed Doppler ultrasound", IEEE trans. on Biomedical Engineering, vol 38, no.6, 1991.

[6] S.Niu, D.Xiao , D.G.Fisher, "A recursive algorithm for simultaneous identification of model order and parameters", IEEE trans. Acoust.,Speech,Signal Processing.,vol 38, pp 884$886,1990$.

[7] S.Niu, D.G.Fisher, D.Xiao:"A factored form of the instrumental variable algorithm", International Journal of adaptive Control and signal processing, vol 7, pp261-273. 1993.

[8] T.Soderstrom and P.Stoica: "Comparaison of some Instrumental Variable Methods - Consistency and Accuracy Aspects", Automatica, vol 17, n 1, pp 101-115, 1981.

[9] G.J.Bierman,C.L.Thornton : $U D U^{T}$ Covariance Factorisation for Kalman filtering". Control and Dynamic Systems, 1980. 